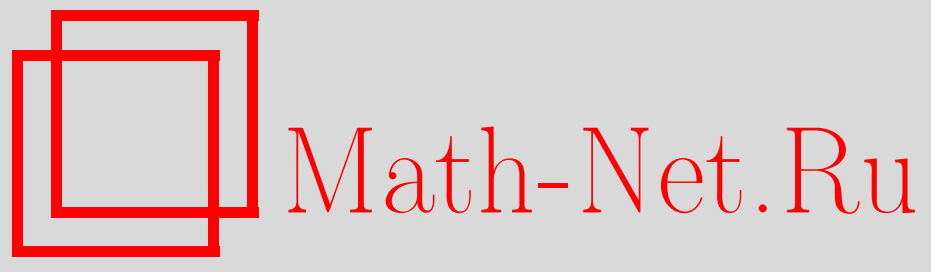

А. В. Буркин, О пороговой вероятности для свойства планарности случайного подграфа регулярного графа, УМH, 2015, том 70, выпуск 6, 205-206

DOI: https://doi.org/10.4213/rm9656

Использование Общероссийского математического портала Math-Net.Ru подразумевает, что вы прочитали и согласны с пользовательским соглашением http://www . mathnet.ru/rus/agreement

Параметры загрузки:

IP : 54.210 .77 .194

26 апреля 2023 г., 13:32:24

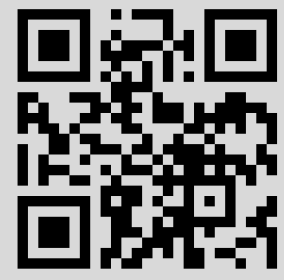




\section{О пороговой вероятности для свойства планарности случайного подграфа регулярного графа}

\section{А. В. Буркин}

1. История задачи и новые результаты. В 60-х годах XX в. П. Эрдёш и А. Реньи предложили модель случайного графа, в котором каждое ребро появляется независимо от остальных ребер с вероятностью $p$. Рассматривается вероятностное пространство $G(n, p)=\left(\Omega_{n}, \mathscr{F}_{n}, \mathrm{P}_{n, p}\right)$, где $\Omega_{n}$ - множество всех неориентированных графов $G=\left(V_{n}, E\right)$ без петель и кратных ребер с множеством вершин $V_{n}=\{1, \ldots, n\}$, $\mathscr{F}_{n}=2^{\Omega_{n}}, \mathrm{P}_{n, p}(G)=p^{|E|}(1-p)^{C_{n}^{2}-|E|}$.

Для $G(n, p)$ был получен ряд результатов, описывающих асимптотические свойства случайного графа при $n \rightarrow \infty$ (см. [1]-[3]).

Определим несколько понятий, используемых в настоящей статье. Обозначим $G=\mathscr{A}$ множество всех графов, обладающих свойством $\mathscr{A}$. Пороговой вероятностъю называется такая функция $p^{*}=p^{*}(n)$, что $\mathrm{P}_{n, p}(G=\mathscr{A}) \rightarrow 0$ при $p=o\left(p^{*}\right), n \rightarrow \infty$, и $\mathrm{P}_{n, p}(G \mid \mathscr{A}) \rightarrow 1$ при $p=w\left(p^{*}\right), n \rightarrow \infty$ (или наоборот). Здесь $f(n)=o(g(n))$ $(f(n)=w(g(n)))$ означает, что для любого $C>0$ существует $n_{0}>0$ такое, что для любого $n>n_{0}$ выполнено $|f(n)|<C|g(n)|(C|g(n)|<|f(n)|)$. Для этих отношений мы также будем использовать обозначения $f(n) \ll g(n)$ и $f(n) \gg g(n)$. Функция $p^{*}=p^{*}(n)$ называется точной пороговой вероятностъю, если $\mathrm{P}_{n, p}(G \models \mathscr{A}) \rightarrow 0$, $n \rightarrow \infty$, при $p \leqslant c p^{*}$, где $c<1$, и $\mathrm{P}_{n, p}(G \mid \mathscr{A}) \rightarrow 1, n \rightarrow \infty$, при $p \geqslant c p^{*}$, где $c>1$ (или наоборот). Будем говорить, что случайный граф обладает свойством $\mathscr{A}$ асимптотически почти наверное (а.п.н.), если $\mathrm{P}_{n, p}(G \models \mathscr{A}) \rightarrow 1$ при $n \rightarrow \infty$.

П. Эрдёш и А. Реньи в [4] доказали, что точной пороговой вероятностью для планарности $G(n, p)$ является функция $p=1 / n$. Их доказательство содержало небольшую ошибку, которая была исправлена позже Т. Лучаком и Дж. Вирманом в [5].

А. Фриз и М. Кривелевич [6] получили следующее обобщение классического утверждения. Пусть $G(n)$ - последовательность конечных графов с минимальной степенью $t=t(n) \rightarrow \infty, n \rightarrow \infty$. Образуем из нее последовательность случайных подграфов $G_{p}=G_{p}(n)$, добавляя в $G_{p}(n)$ каждое ребро $G(n)$ независимо с вероятностью $p=p(n)$.

Теорема 1 (А. Фриз, М. Кривелевич). В указанных обозначениях для любого $\varepsilon>0$ при $p=(1+\varepsilon) / t$ случайный граф $G_{p}$ а.п.н. непланарен.

Будем рассматривать далее последовательности регулярных графов $G(n)$ степени $N_{1}=N_{1}(n)$ с $N_{1} \rightarrow \infty$. Количество вершин графа $G(n)$ будем обозначать $N=N(n)$.

Справедливо следующее несложное утверждение.

ПредЛОЖениЕ 1. Пусть для $G(n)$ выполнено $N \ll N_{1}^{4}$. Тогда при $p \leqslant c / N_{1}$, где $c<1$, случайный граф $G_{p}=G_{p}(n)$ а.п.н. планарен.

Из этого предложения и предыдущей теоремы следует, что при $N \ll N_{1}^{4}$ функция $p=1 / N_{1}$ является точной пороговой вероятностью для свойства планарности.

Рассмотрим случай, когда $N \gg N_{1}^{4}$. Нетрудно показать, что верно следующее утверждение.

ПредлОЖениЕ 2. В указанных условиях функция $p=o\left(\left(1 / N_{1}\right)\left(N_{1}^{4} / N\right)^{1 / 9}\right)$ не может являться пороговой вероятностъю для свойства планарности $G_{p}$.

В то же время в силу теоремы Фриза-Кривелевича пороговая вероятность никогда (с точностью до умножения на константу) не превосходит $1 / N_{1}$.

Работа выполнена при поддержке РФФИ (грант № 15-01-03530).

DOI: $10.4213 / \mathrm{rm} 9656$ 
Интересует вопрос, насколько малой может быть пороговая вероятность. Оказывается, что на некоторых последовательностях графов достигается "нижняя оценка", определенная предложением 2. Сформулируем основной результат.

Теорема 2. Существует такая последовательность регулярных графов $G(n)$ с $N_{1} \rightarrow \infty$ и $\gg N_{1}^{4}$, что пороговая вероятность для свойства планарности $G_{p}(n)$ есть функиия $p^{*}=\left(1 / N_{1}\right)\left(N_{1}^{4} / N\right)^{1 / 9}$. При этом пороговая вероятность не является точной.

2. Идея доказательства. Рассмотрим последовательность графов $G(n, r, s)=$ $(V(n, r), E(n, r, s))$, в которых $V(n, r)=\left\{\mathbf{x}=\left(x_{1}, \ldots, x_{n}\right): x_{i} \in\{0,1\}, x_{1}+\cdots+\right.$ $\left.x_{n}=r\right\}, E(n, r, s)=\{\{\mathbf{x}, \mathbf{y}\}:(\mathbf{x}, \mathbf{y})=s\}$, где $(\mathbf{x}, \mathbf{y})$ обозначает евклидово скалярное произведение.

Такие графы называются дистанционными, поскольку ребра графа соответствуют парам вершин, находящихся на определенном расстоянии друг от друга, в данном случае на расстоянии $\sqrt{2(r-s)}$. Такие графы возникают в задачах комбинаторной геометрии (см. [7]-[13]).

Ясно, что графы $G(n, r, s)$ - регулярные степени $N_{1}=C_{r}^{s} C_{n-r}^{r-s}$ с числом вершин $N=C_{n}^{r}$.

Будем называть случайными дистанционными графами $G_{p}(n, r, s)$ случайные подграфы графов $G(n, r, s)$.

Заметим, что $G_{p}(n, 1,0)=G(n, p)$, т. е. $G_{p}(n, r, s)$ в некотором смысле обобщает классическую модель Эрдёша-Реньи.

Удается доказать теорему о пороговой вероятности для свойства планарности графа $G_{p}(n, r, s)$, из которой следует теорема 2. Напомним, что $f(n) \asymp g(n)$ означает, что существуют такие положительные $n_{1}, C_{1}, C_{2}$, что $C_{1} g(n) \leqslant f(n) \leqslant C_{2} g(n)$ для Bcex $n>n_{1}$.

Теорема 3. Пусть $r, s \in \mathbb{N}$ фиксированы, причем $N_{1}=o\left(N^{1 / 4}\right)$, m.e. $s>3 r / 4$. Тогда пороговой вероятностъю для свойства планарности $G_{p}(n, r, s)$ является функиия $p=\left(1 / N_{1}\right)\left(N_{1}^{4} / N\right)^{1 / 9} \asymp n^{-(6 r-5 s) / 9}$. Кроме того, если $p \sim c n^{-(6 r-5 s) / 9}$, то $\mathrm{P}\left(G_{p}(n, r, s)\right.$ планарен $) \sim \mathrm{P}\left(X_{K_{3,3}}=0\right) \rightarrow e^{-\lambda}$, где $X_{K_{3,3}}$ есть количество $K_{3,3}$ в $G_{p}(n, r, s), a \lambda=c^{9} C_{r}^{s} /\left(72 r !((r-s) !)^{5}\right)$.

Доказательство теоремы 3 использует полученные автором результаты, касающиеся распределения числа копий фиксированных графов в случайных дистанционных графах $G_{p}(n, r, s)$ с постоянными $r$ и $s$.

\section{Список литературы}

[1] B. Bollobás, Random graphs, Cambridge Stud. Adv. Math., 73, Cambridge Univ. Press, Cambridge, 2001, xviii+498 pp. [2] S. Janson, T. Łuczak, A. Ruciński, Random graphs, Wiley-Interscience, New York, 2000, xii+333 pp. [3] В.Ф. Колчин, Случайные графы, Физматлит, M., 2000, 256 c. [4] P. Erdős, A. Rényi, Magyar Tud. Akad. Mat. Kutató Int. Közl., 5 (1960), 17-61. [5] T. Łuczak, J. C. Wierman, Combinatorica, 9:1 (1989), 39-49. [6] A. Frieze, M. Krivelevich, Combin. Probab. Comput., 22:5 (2013), 722-732. [7] А. М. Райгородский, Докл. РАН, 371:5 (2000), 600-603. [8] А. М. Райгородский, УМН, 56:1(337) (2001), 107-146. [9] А. М. Райгородский, Матем. сб., 193:10 (2002), 139-160. [10] А. М. Райгородский, Матем. сб., 196:1 (2005), 123-156. [11] А. М. Райгородский, Изв. РАН. Сер. матем., 69:3 (2005), 81-108. [12] A. M. Raigorodskii, Thirty essays on geometric graph theory, Springer, Berlin, 2013, 429-460. [13] A. M. Raigorodskii, Combinatorica, 32:1 (2012), 111-123.

А. В. Буркин (А. V. Burkin)

Московский государственный университет им. М. В. Ломоносова

E-mail: a.v.burkin@gmail.com
Представлено Ю. С. Ильяшенко Принято редколлегией 12.02.2015 